\section{OPEN ACCESS}

Edited by:

Imtiaz Ahmad Siddiqui,

University of Colorado Anschutz Medical Campus, United States

Reviewed by:

Nidhi Jain,

Cedars-Sinai Medical Center,

United States

Xiangqian Guo,

Henan University, China

*Correspondence:

Hongbing Shen

hbshen@njmu.edu.cn

Hongxia Ma

hongxiama@njmu.edu.cn

tThese authors have contributed equally to this work

Specialty section:

This article was submitted to Cancer Epidemiology and Prevention, a section of the journal Frontiers in Oncology

Received: 09 September 2019 Accepted: 11 December 2019 Published: 15 January 2020

Citation:

Wang L, Zhu M, Wang Y, Fan J, Sun Q, Ji M, Fan X, Xie J, Dai J, Jin G,

Hu Z, Ma H and Shen H (2020)

Cross-Cancer Pleiotropic Analysis Reveals Novel Susceptibility Loci for Lung Cancer. Front. Oncol. 9:1492.

doi: 10.3389/fonc.2019.01492

\title{
Cross-Cancer Pleiotropic Analysis Reveals Novel Susceptibility Loci for Lung Cancer
}

\section{Lijuan Wang ${ }^{1 \dagger}$, Meng Zhu ${ }^{1 \dagger}$, Yuzhuo Wang ${ }^{1}$, Jingyi Fan ${ }^{1}$, Qi Sun ${ }^{1}$, Mengmeng Ji ${ }^{1}$, Xikang Fan ${ }^{1}$, Junxing Xie ${ }^{1}$, Juncheng Dai ${ }^{1,2}$, Guangfu Jin ${ }^{1,2}$, Zhibin Hu ${ }^{1,2}$, Hongxia Ma ${ }^{1,2 *}$ and Hongbing Shen ${ }^{1,2 *}$}

\begin{abstract}
${ }^{1}$ Department of Epidemiology and Biostatistics, Center for Global Health, School of Public Health, Nanjing Medical University, Nanjing, China, ${ }^{2}$ Jiangsu Key Lab of Cancer Biomarkers, Prevention and Treatment, Collaborative Innovation Center for Cancer Medicine, Nanjing Medical University, Nanjing, China
\end{abstract}

Genome-wide association studies (GWASs) have identified hundreds of single nucleotide polymorphisms (SNPs) associated with cancer risk, several of which have shown pleiotropic effects across cancers. Therefore, we performed a systematic cross-cancer pleiotropic analysis to detect the effects of GWAS-identified variants from non-lung cancers on lung cancer risk in 12,843 cases and 12,639 controls from four lung cancer GWASs. The overall association between variants in each cancer and risk of lung cancer was explored using sequential kernel association test (SKAT) analysis. For single variant analysis, we combined the result of specific study using fixed-effect meta-analysis. We performed functional exploration of significant associations based on features from public databases. To further detect the biological mechanism underlying identified observations, pathway enrichment analysis were conducted with R package "clusterProfiler." SNP-set analysis revealed the overall associations between variants of 8 cancer types and lung cancer risk. Single variant analysis identified 6 novel SNPs related to lung cancer risk after multiple correction $\left(P_{\mathrm{fdr}}<0.10\right)$, including rs 1707302 (1p34.1, OR $=0.93,95 \% \mathrm{Cl}$ : 0.90-0.97, $\left.P=7.60 \times 10^{-4}\right)$, rs2516448 (6p21.33, OR =1.07, 95\% Cl: $1.03-1.11, P$ $\left.=1.00 \times 10^{-3}\right), \mathrm{rs} 3869062\left(6 \mathrm{p} 22.1, \mathrm{OR}=0.91,95 \% \mathrm{Cl}: 0.86-0.96, P=7.10 \times 10^{-4}\right)$, rs174549 (11q12.2, OR $\left.=0.90,95 \% \mathrm{Cl}: 0.87-0.94, P=1.00 \times 10^{-7}\right), \mathrm{rs} 7193541$ (16q23.1, OR $\left.=0.93,95 \% \mathrm{Cl}: 0.90-0.96, P=1.20 \times 10^{-4}\right)$, and rs8064454 (17q12, $\left.\mathrm{OR}=1.07,95 \% \mathrm{Cl}: 1.03-1.11, P=4.30 \times 10^{-4}\right)$. The eQTL analysis and functional annotation suggested that these variants might modify lung cancer susceptibility through regulating the expression of related genes. Pathway enrichment analysis showed that genes modulated by these variants play important roles in cancer carcinogenesis. Our findings demonstrate the pleiotropic associations between non-lung cancer susceptibility loci and lung cancer risk, providing important insights into the shared mechanisms of carcinogenesis across cancers.

Keywords: lung cancer, genome-wide association studies, pleiotropy, susceptibility loci, gene expression 


\section{INTRODUCTION}

With rapidly increasing incidence and mortality rates, lung cancer has become the most frequently diagnosed cancer and the leading cause of cancer-related death in recent years. Based on GLOBOCAN 2012, there were 1.82 million new lung cancer cases (12.9\% of the total cancer cases) and 1.59 million deaths (19.4\% of the total cancer deaths) around the world (1). Although tobacco smoking has been confirmed as the main cause of lung cancer, genetic factors also determine lung cancer susceptibility (2). To identify genetic variants that contribute to lung cancer development, several GWASs have been performed and dozens of SNPs were identified over the past few years (3). However, these identified loci could explain only a small fraction of susceptibility. Thus, the challenge remains to detect additional risk loci with small effects, which may partially account for the missing heritability $(4,5)$.

Recent studies have identified that some genetic loci represent pleiotropic associations with multiple cancers (68). For example, genetic variants in the TERT-CLPTM1L region at 5 p15.33 are associated with risk of lung, bladder, prostate, and cervical cancers (8). The discovery of pleiotropic effects may allow for the identification of shared genes and pathways that influence carcinogenesis across different cancers (9). In 2014, Park et al. evaluated the effects of 165 genetic variants associated with non-lung cancers on lung cancer susceptibility, which demonstrated novel susceptibility loci for lung cancer and indicated the commonality between lung cancer and other cancer types (10). However, the number of SNPs in the study by Park et al. was limited and subsequent GWASs have identified more susceptibility loci for cancers in recent years.

Thus, in this study, we comprehensively collected 1,915 GWAS loci associated with non-lung cancers from 5,876 publications and GWAS catalog database, and performed a systematic evaluation of possible pleiotropic associations with lung cancer risk. Our study could provide important insights into pleiotropic associations across cancers and better clarify the mechanism involved in lung cancer susceptibility.

\section{METHODS}

\section{Study Participants}

Data from four existing lung cancer GWASs including 12,843 lung cancer cases and 12,639 controls was used in this study: (i) Nanjing Medical University (NJMU) GWAS including 2,331 lung cancer cases and 3,077 controls (11), (ii) Female Lung Cancer Consortium in Asia (FLCCA) GWAS with 4,796 lung cancer cases and 3,741 controls (12), (iii) Environment and Genetics in Lung Cancer Etiology (EAGLE) GWAS consists of 1,937 lung cancer cases and 1,984 controls and (iv) Division of Cancer Epidemiology and Genetics (DCEG) Lung Cancer GWAS encompasses 3,779 cases and 3,837 controls (13). Briefly, two Asian GWASs and two European GWASs were included in our study to explore the overall genetic effects of variants. The basic demographic information of participants involved are shown in Table 1. Informed consent was obtained from each subject, and this study was approved by the institutional review boards of each participating institution.

\section{Quality Control and Imputation of GWAS Data}

The detail about quality control and imputation has been described in our previous study (11). Briefly, individuals with call rates $<95 \%$, familial relationships or extreme heterozygosity rates were excluded. We selected SNPs based on the following criteria: (i) call rates $>95 \%$, (ii) minor allele frequencies (MAFs) $>0.05$, (iii) $P>1 \times 10^{-6}$ for Hardy-Weinberg equilibrium (HWE). We then phased the haplotypes with Shapeit (14) and performed imputations with IMPUTE2 (15) taken the 1,000 Genomes Project Phase III data as reference. We ruled out SNPs with imputation quality score (INFO) $<0.4$, MAF $<0.01$, and HWE $P<1 \times 10^{-6}$. Quality control procedure was performed using PLINK1.9 software.

\section{SNP Selection}

We undertook a comprehensive systematic review of publications on GWASs and cancers in PubMed using the Mesh Term "Genome-wide association study" or "GWAS" and "cancer." A total of 5,876 abstracts and if necessary the full texts were screened for eligibility. Among them, GWASs, genome-wide meta analyses and replication studies for GWAS loci were evaluated. Additionally, SNPs associated with cancers as of July 2018 from the NHGRI GWAS catalog were also included. Finally, a total of 2,167 SNPs beyond the threshold of significance $\left(P<1 \times 10^{-7}\right)$ remained. After that, we excluded lung cancer GWAS loci as well as those in the same linkage disequilibrium (LD) blocks $\left(r^{2}>0.2\right)$, and 1,915 SNPs within 15 cancer types were remained. The search strategy is shown in Figure 1.

\section{SNP-Set Analysis by Cancer Types}

The aim of SNP-set analysis was to explore the overall associations of variants identified from other cancer types with the risk of lung cancer. SNP-set analysis was conducted using SKAT-C package, which tests for association between groups of SNPs and a phenotype by aggregating the weighted variancecomponent score statistics for each SNP within a group using kernel function (16). In this case, SNPs in $\mathrm{LD}\left(r^{2}>0.2\right)$ with lung cancer GWAS loci were excluded. We divided the remained variants into different groups by cancer types and tested the correlations with lung cancer. Age, gender, smoking status and principal components (PCA) were adjusted in the SNP-set analysis. We used Benjamini-Hochberg method for multiple correction and cancers with FDR less than 0.05 in the combined dataset were considered to be significantly correlated with lung cancer.

\section{Single Variant Analysis}

We used an additive effect model to test the associations of variants with lung cancer in each dataset. Then, meta-analysis was performed using a fixed-effect model. We calculated the index of heterogeneity $\left(I^{2}\right)$ and SNPs with high heterogeneity $\left(I^{2}>75 \%\right)$ were excluded. Then, Benjamini-Hochberg method 
TABLE 1 | Basic characteristics and clinical features of participants in each dataset.

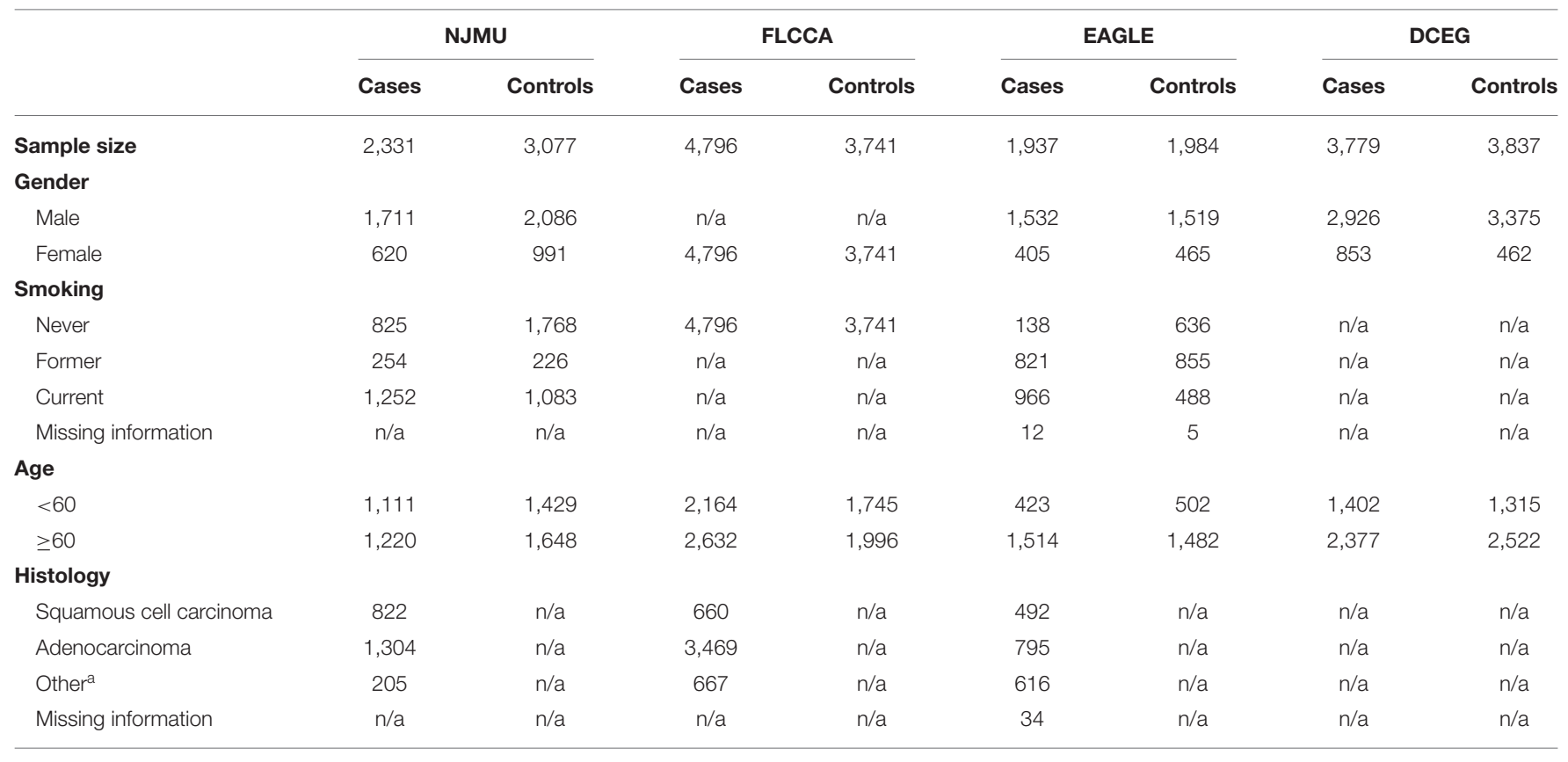

n/a, not available or non-existent.

${ }^{a}$ Other histological types include small cell lung cancer, large cell lung cancer and mixed cell lung cancer.

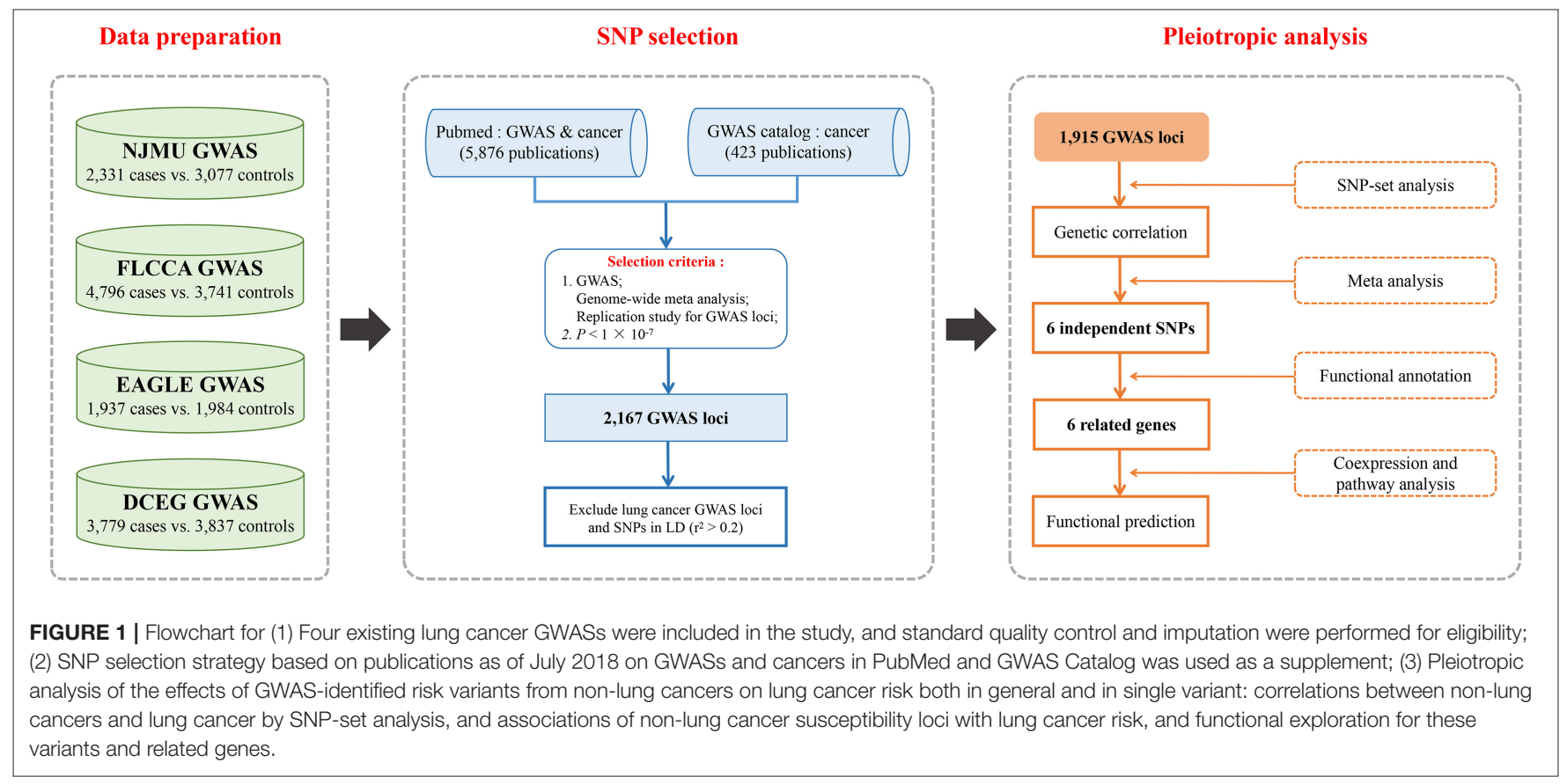

was used to correct multiple testing and FDR less than 0.10 in the combined dataset was considered as a cutoff threshold. In addition, subgroup analyses by age $(\leq 60$ and $>60)$, gender (male and female), smoking status (never and ever), and tumor histology (adenocarcinoma, squamous cell carcinoma and other types of lung cancer) and following gene-environment interaction analysis were performed to further explore the interaction between variants and smoking. Association analyses were conducted with PLINK1.9 while general statistical analyses were carried out using R (R 3.5.0).

\section{Functional Explorations of Significant Associations}

We performed annotations for variants within promising genes using ANNOVAR software (17). We applied the SIFT (18) and PolyPhen databases (19) to predict the function of exonic 
variants. To investigate the potential function of association at non-coding regions, we utilized data from the GenotypeTissue Expression (GTEx, version 7) to perform the expression quantitative trait loci (eQTL) analysis in 383 lung tissue samples. Then, we annotated SNPs to regulatory elements including the histone Chip-seq (H3K27AC, H3K4ME1, H3K4ME3) peaks, DNase I hypersensitivity sites (DHS) and transcription factor binding sites (TFBS) from ENCODE Project Consortium. All these features estimated in A549 cell lines were downloaded from the UCSC website. In addition, we used RegulomeDB database to further evaluate regulatory potential for identified variants.

\section{Co-expression and Pathway Enrichment Analysis}

Based on results from functional annotation, we defined SNPrelated genes with the following criteria: (i) locate within LD blocks where the identified variants as well as their related SNPs $\left(r^{2}>0.6\right)$ reside in, (ii) show most significant cis-eQTL associations with identified variants or bear exonic mutations that affect the function of proteins. In order to test whether these identified related genes were associated with lung cancer susceptibility, we performed gene-based analysis with MAGMA software, which is a powerful tool using multiple regression approach to detect multi-marker effects for a genome-wide gene association analysis (20). To explore biological function and alternative pathways of these related genes, which help to explain their pathogenic mechanism involved in the development of lung cancer, we conducted co-expression and pathway enrichment analysis based on GTEx V7 database and Kyoto Encyclopedia of Genes and Genomes (KEGG, 186 pathways) were used as reference. We used linear regression model to detect coexpressed genes and Bonferroni method was utilized for multiple correction. Significant co-expressed genes with adjusted $P$ value $<0.05$ were included in pathway analysis performed by "clusterProfiler" package (21).

\section{RESULTS}

\section{Correlations Between Non-lung Cancers and Lung Cancer}

In order to obtain a general overview of susceptibility regions for each cancer, we collected the reported GWAS loci and mapped them to particular band of chromosome according to hg19, which was the susceptibility band we mentioned below. To date, 60 susceptibility bands have been identified for lung cancer and 50 of them were overlapped with the bands of nonlung cancer (Figure 2), further suggesting that some genomic bands are associated with multiple cancers. Here, we defined susceptibility bands shared by more than half of cancers (i.e., shared by at least 8 cancer types) as cancer enriched bands, and 4 (5p15.33, 6p21.32, 8q24.21, 9p21.3) met the criterion and showed pleiotropic associations with multiple cancers. Supplementary Table 1 displayed an overview of GWAS loci located in cancer enriched bands identified in different cancers, some of which showed significant associations with lung cancer risk, indicating the correlations between non-lung cancers and lung cancer.

In the SNP-set analysis, we found that the GWAS SNPs of 8 cancer types, including, cervical/endometrial, bladder/renal, prostate, pancreatic, ovarian, leukemia/lymphoma, esophageal, and colorectal cancer, were significantly associated with

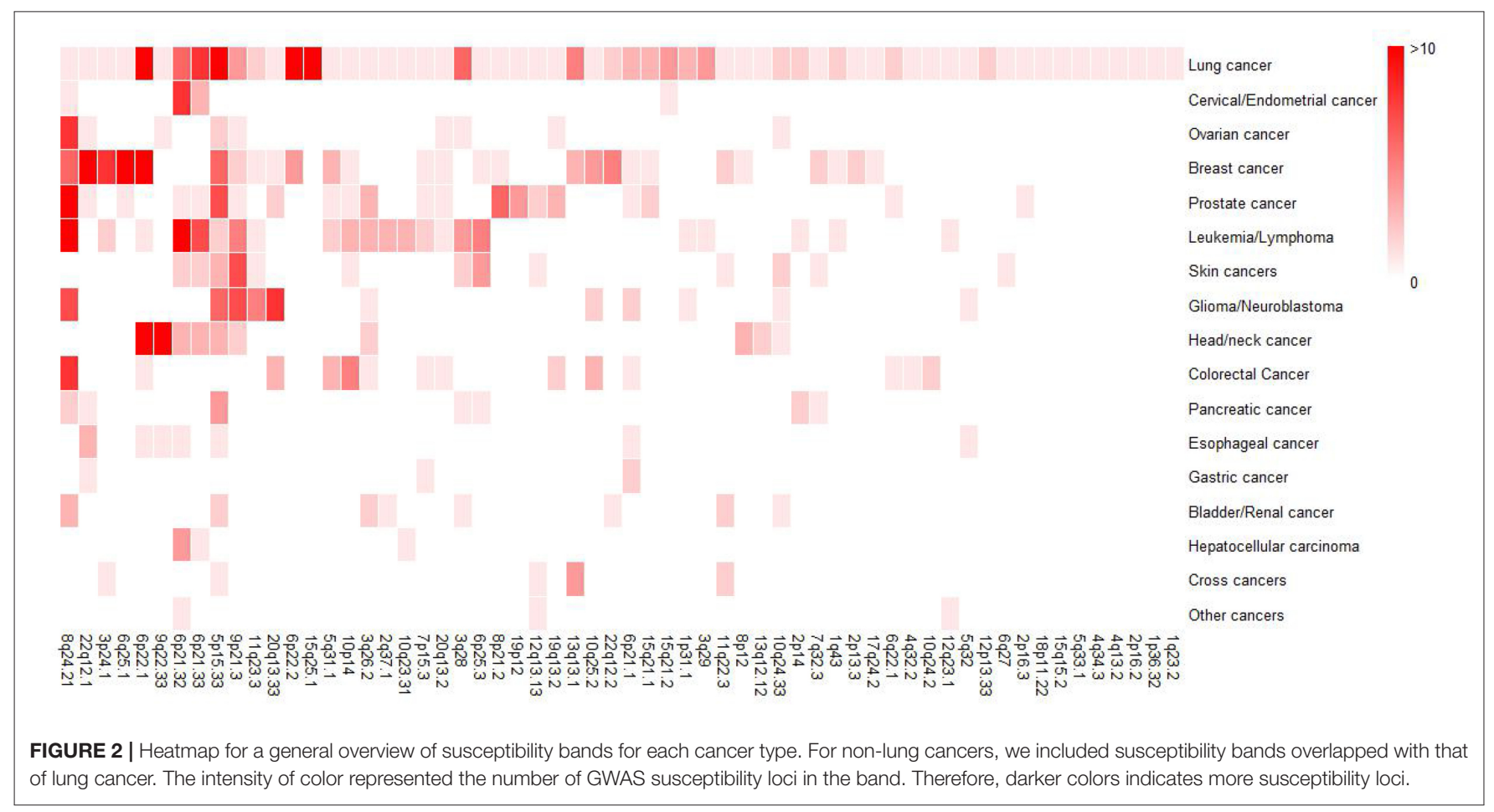


lung cancer risk in the combined dataset (FDR $<0.05$ ) (Supplementary Table 2). To rule out the impact of shared bands among different cancers (i.e., MHC), we excluded variants within MHC as well as the 4 cancer enriched bands mentioned above and re-conducted a SNP-set analysis. Similar associations were observed in cervical/endometrial, bladder/renal, prostate, ovarian, leukemia/lymphoma, esophageal, and colorectal cancer, which were still correlated with lung cancer (Supplementary Table 3).

\section{Single Variant Analysis and Subgroup Analysis}

After combining results from four GWAS datasets of lung cancer, we identified 17 SNPs that were significantly related to lung cancer susceptibility $\left(P_{\text {fdr }}<0.10\right)$ (Supplementary Table 4). For variants in the same LD block $\left(r^{2}>0.2\right)$, the most significant one remained and 6 independent SNPs were showed in Table 2. Supplementary Table 5 displays the association results of these 6 variants in each separate cohort. The regional plots of these 6 variants were presented in Supplementary Figure 1. Among these 6 identified novel susceptibility loci, rs2516448 (6p21.33, $\left.\mathrm{OR}=1.07,95 \% \mathrm{CI}: 1.03-1.11, P=1.00 \times 10^{-3}\right)$ and $\mathrm{rs} 3869062$ $\left(6 \mathrm{p} 22.1, \mathrm{OR}=0.91,95 \% \mathrm{CI}: 0.86-0.96, P=7.10 \times 10^{-4}\right)$ reside in known lung cancer susceptibility regions, but were independent from previously reported SNPs of lung cancer (Supplementary Table 6); while rs1707302 (1p34.1, OR = 0.93, 95\% CI: $\left.0.90-0.97, P=7.60 \times 10^{-4}\right)$, rs174549 (11q12.2, OR = 0.90, 95\% CI: $\left.0.87-0.94, P=1.00 \times 10^{-7}\right)$, rs7193541 (16q23.1, OR $=0.93,95 \%$ CI: $\left.0.90-0.96, P=1.20 \times 10^{-4}\right)$ and $\mathrm{rs} 8064454$ $\left(17 \mathrm{q} 12\right.$, OR $\left.=1.07,95 \% \mathrm{CI}: 1.03-1.11, P=4.30 \times 10^{-4}\right)$ were located in novel susceptibility bands for lung cancer and were firstly identified to be correlated with lung cancer risk in this study.

Subgroup analyses were conducted according to age, gender, smoking status, and tumor histology to investigate potential large differences among subgroups of population. As shown in Figure 3, the association for rs3869062 showed heterogeneous among different tumor histology $\left(P_{\text {heterogeneity }}<0.01\right)$, where the association appeared to be significant to adenocarcinoma (OR $=0.91 ; 95 \%$ CI: $0.85-0.97)$ but not squamous cell carcinoma $(\mathrm{OR}=1.08 ; 95 \% \mathrm{CI}: 0.99-1.19)$. We also identify significant heterogeneity among different tumor histology in the association for $\operatorname{rs} 174549\left(P_{\text {heterogeneity }}=0.02\right)$, where other histological types include small cell lung cancer and large cell lung cancer show more significant association with lung cancer susceptibility $(\mathrm{OR}=0.82 ; 95 \% \mathrm{CI}$ : 0.76-0.89). Besides, rs8064454 showed a significant association with lung cancer risk in non-smokers ( $P_{\text {heterogeneity }}<0.01$, OR $=0.88,95 \%$ CI: $\left.0.83-0.93\right)$, while not in smokers (OR $=1.00$; 95\% CI: 0.93-1.07). Then, a significant interaction $(P$-interaction $=0.01$, Supplementary Table 7$)$ was observed between rs8064454 and smoking.

\section{Functional Annotation and Pathway Enrichment Analysis}

To further unearth the underlying biological function of identified SNPs, we carried out a systematic functional annotation analysis in silico. The identified variants as well as their high $\operatorname{LD}\left(r^{2}>0.6\right)$ variants were evaluated in our study. Among these variants, four were missense but none was predicted as deleterious (Supplementary Table 8). Based on results from GTEx, we found that the G allele of rs1707302 was significantly associated with increased expression of MAST2 ( $\beta$ $=0.16, P=4.30 \times 10^{-7}$, Supplementary Figure 2A). Besides, rs3869062 and rs8064454 showed significant associations with up-regulated $H L A-G\left(\beta=0.77, P=9.60 \times 10^{-8}\right.$, Supplementary Figure 2B) and $\operatorname{HNF} 1 B(\beta=0.065, P=$ 0.028, Supplementary Figure 2C), respectively. While the protective allele of $\mathrm{rs} 2516448$ and rs7193541 were related to decreased expression of MICA $(\beta=-0.34, P=1.50 \times$ $10^{-14}$, Supplementary Figure 2D) and RFWD3 $(\beta=-0.23$, $P=2.20 \times 10^{-7}$, Supplementary Figure 2E). The rs174549 was in high LD with rs174548 $\left(r^{2}=0.98\right)$, which was proven to be correlated with lung cancer risk by regulating FADS1 gene expression in liver tissues $\left(\beta=-0.23, P=2.20 \times 10^{-7}\right.$, Supplementary Figure 2F) and plasma levels of polyunsaturated fatty acids (PUFAs) according to our recent study (22).

Gene-based analysis revealed that the associations between identified related genes and lung cancer risk were statistical significant $(P<0.05$, Supplementary Table 9). To further explore the biological process of these identified related genes, we performed co-expressed analysis using data from GTEx V7 database and implemented pathway analysis with KEGG database. At the level of statistical significance $\left(P_{\mathrm{fdr}}<\right.$ $0.05)$, related genes along with their co-expressed genes were significantly aggregated in cancer associated pathways, such as p53 signaling pathway (23), Ras signaling pathway (24), MAPK signaling pathway (Supplementary Table 10) (25).

TABLE 2 | Independent associations of significant locus with lung cancer risk.

\begin{tabular}{|c|c|c|c|c|c|c|c|}
\hline Band & Related gene & SNP & EA/NEA & OR $(95 \% \mathrm{CI})^{\mathrm{a}}$ & $P^{a}$ & FDR $^{a}$ & Source of Cancer \\
\hline $1 p 34.1$ & MAST2 & rs1707302 & $A / G$ & 0.93(0.90-0.97) & 7.60E-04 & 6.60E-02 & Breast cancer \\
\hline $6 p 21.33$ & MICA & rs2516448 & $\mathrm{T} / \mathrm{C}$ & 1.07(1.03-1.11) & 1.00E-03 & 8.10E-02 & Cervical cancer \\
\hline $6 p 22.1$ & HLA-G & rs3869062 & $\mathrm{G} / \mathrm{A}$ & $0.91(0.86-0.96)$ & 7.10E-04 & 6.60E-02 & Nasopharyngeal carcinoma \\
\hline $11 \mathrm{q} 12.2$ & FADS1 & rs174549 & $A / G$ & $0.90(0.87-0.94)$ & 1.00E-07 & $1.40 \mathrm{E}-04$ & Laryngeal squamous cell carcinoma \\
\hline $16 q 23.1$ & RFWD3 & rs7193541 & $\mathrm{C} / \mathrm{T}$ & $0.93(0.90-0.96)$ & $1.20 \mathrm{E}-04$ & $1.90 \mathrm{E}-02$ & Multiple myeloma \\
\hline $17 q 12$ & HNF1B & rs8064454 & $\mathrm{C} / \mathrm{A}$ & 1.07(1.03-1.11) & 4.30E-04 & 5.70E-02 & Prostate cancer \\
\hline
\end{tabular}

EA, effect allele; NEA, non-effect allele.

a Based on meta-analysis of logistic regression results from 4 lung cancer GWASs. 
A

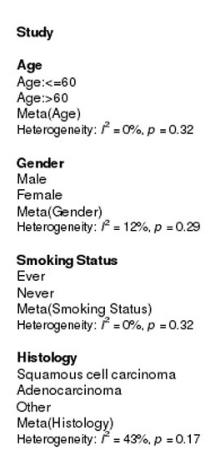

C
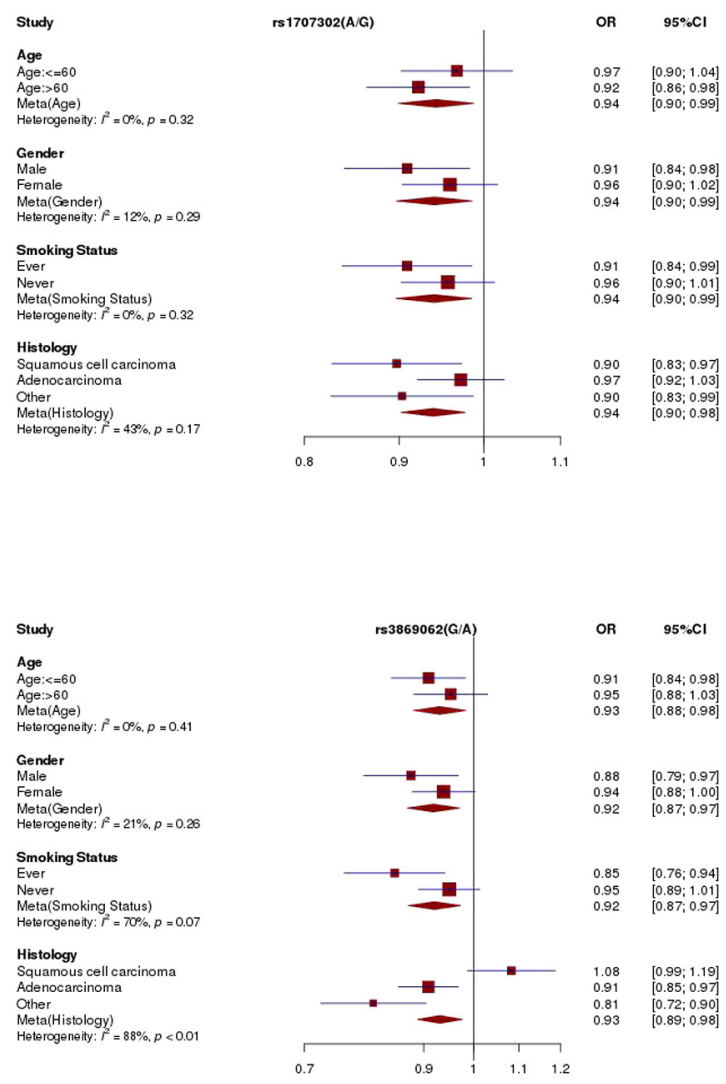

$\mathbf{E}$

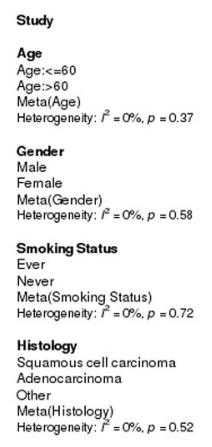

B
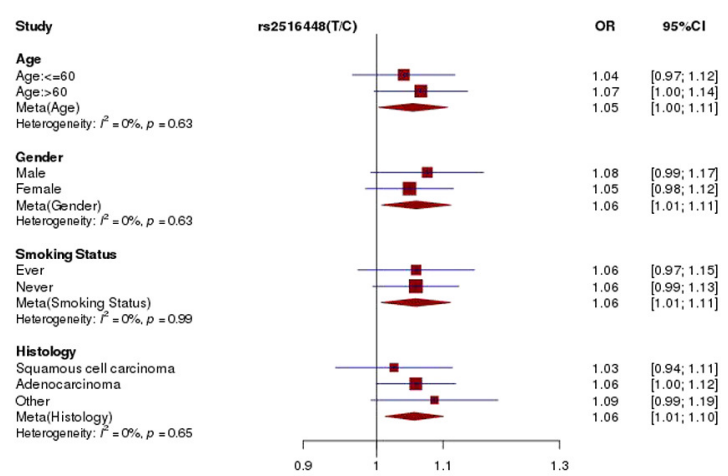

D
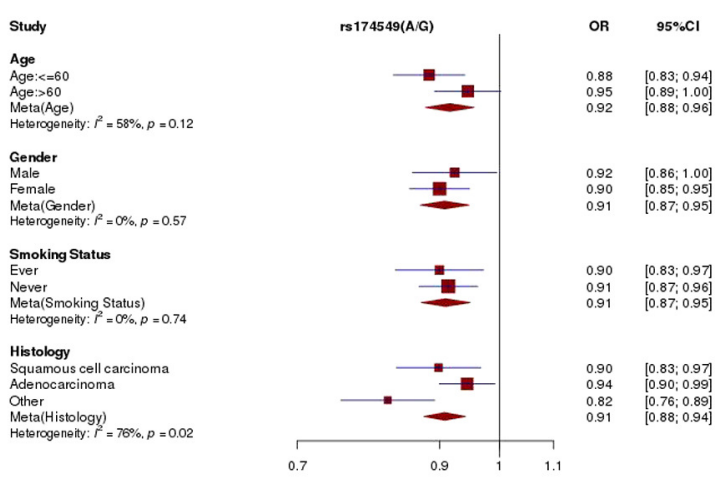

\section{F}

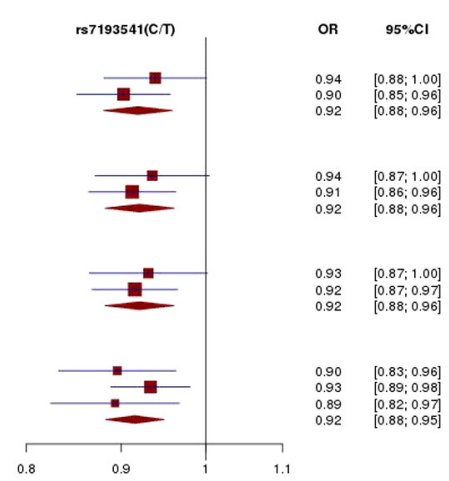

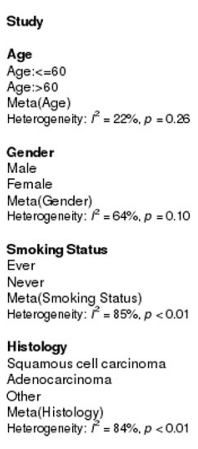

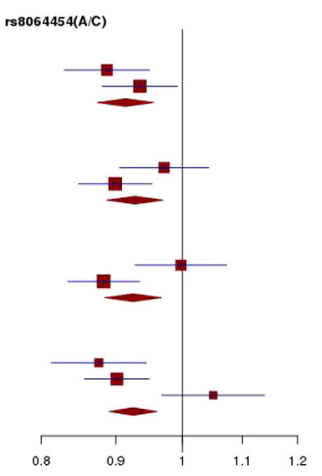

0.99

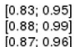

$9.97 \quad[0.91: 1.04$

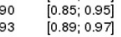

$\begin{array}{ll}1.00 & {[0.931 .07]} \\ 0.88 & 0.93 \\ 0.93 & 0.83 \\ {[0.88} & 0.97]\end{array}$

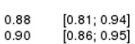

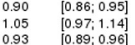

FIGURE 3 | The forest plot of 6 significant SNPs. (A) The forest plot of rs1707302. (B) The forest plot of rs2516448. (C) The forest plot of rs3869062. (D) The forest plot of rs174549. (E) The forest plot of rs7193541. (F) The forest plot of rs8064454.

\section{DISCUSSION}

In our study, we performed a pleiotropic analysis to explore the shared susceptibility mechanisms between non-lung cancers and lung cancer and found genetic variants identified from other cancer types were also significantly associated with the risk of lung cancer. In the single variant analysis, 6 novel susceptibility loci were identified related to lung cancer susceptibility after multiple corrections. Functional exploration revealed that these variants may modulate lung cancer risk by regulating the expression of related genes, which play important role in cancer development. Our findings demonstrate shared genetic components across cancers and provide important insight for future investigation of biological mechanisms involved in cancer progression.

We conduct a systematic scan on the susceptibility bands for each cancer and found that the majority (50/60) of lung cancer susceptibility bands are in common with that of non-lung 
cancers. Besides, pleiotropic analysis showed that previously reported GWAS SNPs for non-lung cancer were also associated with the risk of lung cancer, indicating shared heritability across cancers. However, for the lack of GWAS summary data of each cancer, which is necessary for estimating the correlation explained by the effects of common SNPs, we performed a SNP-set analysis based on our existing lung cancer GWAS data, and identified 8 cancer types correlated with lung cancer. Some previous studies also reported similar findings (26-29). Lindström et al. found significant genetic correlation between colorectal and lung cancer $\left(r_{\mathrm{g}}=0.31\right)(26)$; Sampson et al. identified that bladder cancer was statistically correlated with lung cancer $\left(r_{\mathrm{g}}=0.35\right)$ (27); Recently, Xia et al. revealed significant genetic correlation between ovarian and lung cancer $\left(r_{\mathrm{g}}=0.18\right)(28)$. Additionally, the genetic associations based on UK Biobank demonstrated that lung cancer was genetically correlated with blood cell tumors $\left(r_{\mathrm{g}}=0.18\right)$ and malignant neoplasms of digestive organs $\left(r_{\mathrm{g}}=0.10\right)$ (29). Our data supported these findings, and identify another 2 cancer types (cervical and prostate cancer) correlated with lung cancer. The mechanism underlying these observations has not been wellcharacterized. It is likely that the correlations are due to the shared genetic factors such as penetrant mutations in BRCA2, which predisposes to breast, ovarian, lung, and prostate cancers $(30,31)$. In addition, the correlations between ovarian, colorectal and lung cancer might be driven in part by genetic variants in the inflammation pathway (32). Interestingly, bladder cancer was identified to be genetic correlated with both lung cancer and smoking (27), suggesting the overlapped smoking-related SNPs among these cancers.

Rs1707302 (1p34.1), a known risk locus of breast cancer, was associated with the risk of lung cancer in our study. SNPs in LD $\left(r^{2}>0.6\right)$ with $r s 1707302$ mainly locate in the functional regions of MAST2 (Supplementary Table 7), which encodes a microtubule-associated serine/threonine kinase. MAST2 has been identified to be involved in PI3K-AKT signaling pathway $(33,34)$, which plays crucial role in regulating many cellular processes including cell proliferation, survival, growth and motility (35). Consistent with these findings, we found that genes co-expressed with MAST2 were significantly enriched in cell metabolism, DNA replication and RNA polymerase pathways.

For $6 \mathrm{p} 21.33$, we identified that the cervical cancer susceptibility locus, rs2516448, was associated with lung cancer susceptibility. This variant was related to the expression of $M I C A$, a stress-induced gene expressed by cancer cells, function as ligands for NKG2D receptors (36). Proteolytic shedding of MICA from tumor cells, might promote immunosubversion by reducing the expression of NKG2D (37). Besides, soluble MICA released by tumor cells contributes to tumor immune evasion through down-regulating NKG2D and inactiving tumor-antigen-specific effector T cells (38). Chen et al. reported that rs2516448 is in perfect LD with a frameshift mutation (A5.1) in MICA exon 5, which results in less membrane-bound $M I C A$, causing immune inactivation and tumor progression (39). Furthermore, pathway analysis revealed an enrichment of genes co-expressed with MICA in Non-small cell lung cancer,
Ras signaling pathway, MAPK signaling pathway, etc, indicating the important role of MICA in carcinogenesis process.

Two head/neck cancer GWAS SNP, rs3869062 (6p22.1) and rs174549 (11q12.2), were correlated with lung cancer susceptibility. Rs3869062 showed significant association with increased expression of $H L A-G$, which has been reported to be involved in immune recognition and might manipulate tumor specific immune responses through cytokine production (40). For rs174549, a recent metabolome-wide association study identified its high LD $\left(r^{2}=0.98\right)$ variant, rs174548, associated with both plasma levels of polyunsaturated fatty acids (PUFAs) and lung cancer risk, proposing that plasma PUFAs might function as risk indicator of lung cancer (22). However, challenge remains to unravel the molecular mechanisms underlying observations and further investigations are needed.

The multiple myeloma susceptibility locus, rs7193541, was located in the exon of RFWD3 in 16q23.1. Functional annotation revealed that rs7193541 is a missense variant of $R F W D 3$ and its related SNPs $\left(r^{2}>0.6\right)$ mainly locate in the functional regions of RFWD3. RFWD3 is an E3 ubiquitin ligase that involves in replication protein A (RPA) mediated DNA damage and repair (41). In addition, it has been suggested that RFWD3 mediates the ubiquitination of p53/TP53 by forming a RFWD3-MDM2-p53 complex in the late response to DNA damage (42). Consistent with previous observations $(41,42)$, genes co-expressed with RFWD3 were enriched in Cell cycle, DNA replication and p53 signaling pathway, suggesting that RFWD3 contributes to lung cancer susceptibility possibly by manipulating DNA repair process.

The rs8064454, a previously reported susceptibility loci for prostate cancer, is located in the intron of $H N F 1 B$ at chromosome $17 q 12$. Rs8064454 was associated with the expression of HNF1B and variants in $\operatorname{LD}\left(r^{2}>0.6\right)$ with $\mathrm{rs} 8064454$ all reside in a region that has a chromatin state indicative of promoter and enhancer elements. $H N F 1 B$, a member of the homeodomaincontaining superfamily of transcription factors (TFs), has been demonstrated to act as a bookmarking factor and bind to mitotic chromatin with efficient DNA binding ability, playing crucial roles in the epigenetic transmission of information through the cell cycle (43). Thus, abnormal mitotic chromatin binding induced by mutations of $H N F 1 B$ may be responsible for human pathological conditions. For instance, SNPs of HNF1B are associated with risk of multiple cancers, including prostate (44), ovarian (45), endometrial (46), and renal cell carcinomas (47). Our study found that genes co-expressed with $H N F 1 B$ were correlated with Ras signaling pathway, EGFR tyrosine kinase inhibitor resistance, mTOR signaling pathway, etc.

In conclusion, we systematically evaluated the association of non-lung cancer susceptibility loci with lung cancer risk. We also identified 6 independent SNPs associated with the susceptibility to lung cancer. Functional exploration revealed that the related genes may be involved in cancer-associated pathways across multiple cancers. However, one limitation of our study is that we lack detailed biological mechanisms of how these SNPs are associated with different pathways and thus are involved in different cancers. In this case, molecular experiments are 
warranted to better characterize the effects of identified variants as well as related genes on lung cancer development.

\section{DATA AVAILABILITY STATEMENT}

SNP data is available in the FigShare database (https://figshare. com/), doi: https://dx.doi.org/10.6084/m9.figshare.11346668.

\section{ETHICS STATEMENT}

The studies involving human participants were reviewed and approved by Ethics Committee of Nanjing Medical University. The patients/participants provided their written informed consent to participate in this study.

\section{AUTHOR CONTRIBUTIONS}

HS, HM, ZH, GJ, and JD: study conception and design and manuscript review. YW, JF, QS, and MJ: literature review and data extraction and quality control. LW, XF, and JX: statistical analysis. LW and MZ: manuscript preparation.

\section{REFERENCES}

1. Torre LA, Bray F, Siegel RL, Ferlay J, Lortet-Tieulent J, Jemal A. Global cancer statistics, 2012. CA Cancer J Clin. (2015) 65:87-108. doi: 10.3322/caac.21262

2. Lichtenstein P, Holm NV, Verkasalo PK, Iliadou A, Kaprio J, Koskenvuo M, et al. Environmental and heritable factors in the causation of cancer-analyses of cohorts of twins from Sweden, Denmark, and Finland. N Engl J Med. (2000) 343:78-85. doi: 10.1056/NEJM200007133430201

3. Bosse Y, Amos CI. A decade of GWAS results in lung cancer. Cancer Epidemiol Biomarkers Prev. (2018) 27:363-79. doi: 10.1158/1055-9965.EPI-16-0794

4. Manolio TA, Collins FS, Cox NJ, Goldstein DB, Hindorff LA, Hunter DJ, et al. Finding the missing heritability of complex diseases. Nature. (2009) 461:747-53. doi: 10.1038/nature08494

5. Hindorff LA, Sethupathy P, Junkins HA, Ramos EM, Mehta JP, Collins FS, et al. Potential etiologic and functional implications of genome-wide association loci for human diseases and traits. Proc Natl Acad Sci USA. (2009) 106:9362-7. doi: 10.1073/pnas.0903103106

6. Ghoussaini M, Song H, Koessler T, Al Olama AA, Kote-Jarai Z, Driver $\mathrm{KE}$, et al. Multiple loci with different cancer specificities within the 8q24 gene desert. J Natl Cancer Inst. (2008) 100:962-6. doi: 10.1093/jnci/ $\operatorname{djn} 190$

7. Jin G, Ma H, Wu C, Dai J, Zhang R, Shi Y, et al. Genetic variants at $6 \mathrm{p} 21.1$ and 7 p15.3 are associated with risk of multiple cancers in Han Chinese. Am J Hum Genet. (2012) 91:928-34. doi: 10.1016/j.ajhg.2012.09.009

8. Rafnar T, Sulem P, Stacey SN, Geller F, Gudmundsson J, Sigurdsson A, et al. Sequence variants at the TERT-CLPTM1L locus associate with many cancer types. Nat Genet. (2009) 41:221-7. doi: 10.1038/ng.296

9. Fehringer G, Kraft P, Pharoah PD, Eeles RA, Chatterjee N, Schumacher FR, et al. Cross-cancer genome-wide analysis of lung, ovary, breast, prostate, and colorectal cancer reveals novel pleiotropic associations. Cancer Res. (2016) 76:5103-14. doi: 10.1158/0008-5472.CAN-15-2980

10. Park SL, Fesinmeyer MD, Timofeeva M, Caberto CP, Kocarnik JM, Han Y, et al. Pleiotropic associations of risk variants identified for other cancers with lung cancer risk: the PAGE and TRICL consortia. J Natl Cancer Inst. (2014) 106:dju061. doi: 10.1093/jnci/dju061

11. Hu Z, Wu C, Shi Y, Guo H, Zhao X, Yin Z, et al. A genome-wide association study identifies two new lung cancer susceptibility loci at 13q12.12 and 22q12.2 in Han Chinese. Nat Genet. (2011) 43:792-6. doi: 10.1038/ng.875

\section{FUNDING}

This work was funded by the National Key Research and Development Program of China (2017YFC0907905, 2018YFC1315002-1), National Natural Science of China (81922061, 81973123, 81521004, and 81803306), Natural Science Foundation of Jiangsu Province (BK20180675), the Natural Science Foundation of the Jiangsu Higher Education Institutions of China (18KJB330002), and National Science Foundation for Post-doctoral Scientists of China (2018M640466).

\section{ACKNOWLEDGMENTS}

We are very grateful to all teachers and students involved in this study for their contributions and helpful comments.

\section{SUPPLEMENTARY MATERIAL}

The Supplementary Material for this article can be found online at: https://www.frontiersin.org/articles/10.3389/fonc. 2019.01492/full\#supplementary-material

12. Lan Q, Hsiung CA, Matsuo K, Hong YC, Seow A, Wang Z, et al. Genome-wide association analysis identifies new lung cancer susceptibility loci in neversmoking women in Asia. Nat Genet. (2012) 44:1330-5. doi: 10.1038/ng.2456

13. Landi MT, Chatterjee N, Yu K, Goldin LR, Goldstein AM, Rotunno M, et al. A genome-wide association study of lung cancer identifies a region of chromosome 5p15 associated with risk for adenocarcinoma. Am J Hum Genet. (2009) 85:679-91. doi: 10.1016/j.ajhg.2009.09.012

14. Delaneau O, Marchini J, Zagury JF. A linear complexity phasing method for thousands of genomes. Nat Methods. (2011) 9:179-81. doi: 10.1038/nmeth.1785

15. Howie BN, Donnelly P, Marchini J. A flexible and accurate genotype imputation method for the next generation of genome-wide association studies. PLoS Genet. (2009) 5:e1000529. doi: 10.1371/journal.pgen.1000529

16. Ionita-Laza I, Lee S, Makarov V, Buxbaum JD, Lin X. Sequence kernel association tests for the combined effect of rare and common variants. Am J Hum Genet. (2013) 92:841-53. doi: 10.1016/j.ajhg.2013.04.015

17. Wang K, Li M, Hakonarson H. ANNOVAR: functional annotation of genetic variants from high-throughput sequencing data. Nucleic Acids Res. (2010) 38:e164. doi: 10.1093/nar/gkq603

18. Ng PC, Henikoff S. SIFT: predicting amino acid changes that affect protein function. Nucleic Acids Res. (2003) 31:3812-4. doi: 10.1093/nar/gkg509

19. Adzhubei IA, Schmidt S, Peshkin L, Ramensky VE, Gerasimova A, Bork P, et al. A method and server for predicting damaging missense mutations. Nat Methods. (2010) 7:248-9. doi: 10.1038/nmeth0410-248

20. de Leeuw CA, Mooij JM, Heskes T, Posthuma D. MAGMA: generalized gene-set analysis of GWAS data. PLoS Comput Biol. (2015) 11:e1004219. doi: 10.1371/journal.pcbi.1004219

21. Yu G, Wang LG, Han Y, He QY. clusterProfiler: an R package for comparing biological themes among gene clusters. OMICS. (2012) 16:284-7. doi: 10.1089/omi.2011.0118

22. Wang C, Qin N, Zhu M, Chen M, Xie K, Cheng Y, et al. Metabolomewide association study identified the association between a circulating polyunsaturated fatty acids variant rs 174548 and lung cancer. Carcinogenesis. (2017) 38:1147-54. doi: 10.1093/carcin/bgx084

23. Toledo F, Wahl GM. Regulating the 553 pathway: in vitro hypotheses, in vivo veritas. Nat Rev Cancer. (2006) 6:909-23. doi: 10.1038/nrc2012

24. Kumar MS, Hancock DC, Molina-Arcas M, Steckel M, East P, Diefenbacher $\mathrm{M}$, et al. The GATA2 transcriptional network is requisite for RAS 
oncogene-driven non-small cell lung cancer. Cell. (2012) 149:642-55. doi: 10.1016/j.cell.2012.02.059

25. Wagner EF, Nebreda AR. Signal integration by JNK and p38 MAPK pathways in cancer development. Nat Rev Cancer. (2009) 9:537-49. doi: $10.1038 / \mathrm{nrc} 2694$

26. Lindstrom S, Finucane H, Bulik-Sullivan B, Schumacher FR, Amos CI, Hung RJ, et al. Quantifying the genetic correlation between multiple cancer types. Cancer Epidemiol Biomarkers Prev. (2017) 26:1427-35. doi: 10.1158/1055-9965.EPI-17-0211

27. Sampson JN, Wheeler WA, Yeager M, Panagiotou O, Wang Z, Berndt SI, et al. Analysis of heritability and shared heritability based on genome-wide association studies for thirteen cancer types. J Natl Cancer Inst. (2015) 107:djv279. doi: 10.1093/jnci/djv279

28. Jiang X, Finucane HK, Schumacher FR, Schmit SL, Tyrer JP, Han Y, et al. Shared heritability and functional enrichment across six solid cancers. Nat Commun. (2019) 10:431. doi: 10.1038/s41467-018-08054-4

29. Canela-Xandri O, Rawlik K, Tenesa A. An atlas of genetic associations in UK Biobank. Nat Genet. (2018) 50:1593-9. doi: 10.1038/s41588-018-0248-z

30. Maxwell KN, Domchek SM. Cancer treatment according to BRCA1 and BRCA2 mutations. Nat Rev Clin Oncol. (2012) 9:520-8. doi: $10.1038 /$ nrclinonc. 2012.123

31. Wang Y, McKay JD, Rafnar T, Wang Z, Timofeeva MN, Broderick P, et al. Rare variants of large effect in BRCA2 and CHEK2 affect risk of lung cancer. Nat Genet. (2014) 46:736-41. doi: 10.1038/ng.3002

32. Hung RJ, Ulrich CM, Goode EL, Brhane Y, Muir K, Chan AT, et al. Cross cancer genomic investigation of inflammation pathway for five common cancers: lung, ovary, prostate, breast, and colorectal cancer. J Natl Cancer Inst. (2015) 107:djv246. doi: 10.1093/jnci/djv246

33. Valiente M, Andres-Pons A, Gomar B, Torres J, Gil A, Tapparel C, et al. Binding of PTEN to specific PDZ domains contributes to PTEN protein stability and phosphorylation by microtubule-associated serine/threonine kinases. J Biol Chem. (2005) 280:28936-43. doi: 10.1074/jbc.M5047 61200

34. Lee JO, Yang H, Georgescu MM, Di Cristofano A, Maehama T, Shi Y, et al. Crystal structure of the PTEN tumor suppressor: implications for its phosphoinositide phosphatase activity and membrane association. Cell. (1999) 99:323-34.

35. Vivanco I, Sawyers CL. The phosphatidylinositol 3-Kinase AKT pathway in human cancer. Nat Rev Cancer. (2002) 2:489-501. doi: 10.1038/nrc839

36. Dranoff G. Cytokines in cancer pathogenesis and cancer therapy. Nat Rev Cancer. (2004) 4:11-22. doi: 10.1038/nrc1252

37. Zitvogel L, Tesniere A, Kroemer G. Cancer despite immunosurveillance: immunoselection and immunosubversion. Nat Rev Immunol. (2006) 6:71527. doi: $10.1038 /$ nri1936
38. Groh V, Wu J, Yee C, Spies T. Tumour-derived soluble MIC ligands impair expression of NKG2D and T-cell activation. Nature. (2002) 419:734-8. doi: 10.1038/nature01112

39. Chen D, Juko-Pecirep I, Hammer J, Ivansson E, Enroth S, Gustavsson I, et al. Genome-wide association study of susceptibility loci for cervical cancer. J Natl Cancer Inst. (2013) 105:624-33. doi: 10.1093/jnci/djt051

40. Seliger B, Abken H, Ferrone S. HLA-G and MIC expression in tumors and their role in anti-tumor immunity. Trends Immunol. (2003) 24:82-7. doi: 10.1016/s1471-4906(02)00039-x

41. Feeney L, Munoz IM, Lachaud C, Toth R, Appleton PL, Schindler D, et al. RPA-mediated recruitment of the E3 ligase RFWD3 is vital for interstrand crosslink repair and human health. Mol Cell. (2017) 66:610-21 e4. doi: 10.1016/j.molcel.2017.04.021

42. Fu X, Yucer N, Liu S, Li M, Yi P, Mu JJ, et al. RFWD3-Mdm2 ubiquitin ligase complex positively regulates p53 stability in response to DNA damage. Proc Natl Acad Sci USA. (2010) 107:4579-84. doi: 10.1073/pnas.0912094107

43. Lerner J, Bagattin A, Verdeguer F, Makinistoglu MP, Garbay S, Felix T, et al. Human mutations affect the epigenetic/bookmarking function of HNF1B. Nucleic Acids Res. (2016) 44:8097-111. doi: 10.1093/nar/gkw467

44. Sun J, Zheng SL, Wiklund F, Isaacs SD, Purcell LD, Gao Z, et al. Evidence for two independent prostate cancer risk-associated loci in the HNF1B gene at 17q12. Nat Genet. (2008) 40:1153-5. doi: 10.1038/ng.214

45. Pharoah PD, Tsai YY, Ramus SJ, Phelan CM, Goode EL, Lawrenson $\mathrm{K}$, et al. GWAS meta-analysis and replication identifies three new susceptibility loci for ovarian cancer. Nat Genet. (2013) 45:362-70, 370e1-2. doi: $10.1038 / \mathrm{ng} .2564$

46. Spurdle AB, Thompson DJ, Ahmed S, Ferguson K, Healey CS, O'Mara T, et al. Genome-wide association study identifies a common variant associated with risk of endometrial cancer. Nat Genet. (2011) 43:451-4. doi: 10.1038/ng.812

47. Rebouissou S, Vasiliu V, Thomas C, Bellanne-Chantelot C, Bui H, Chretien Y, et al. Germline hepatocyte nuclear factor 1alpha and 1beta mutations in renal cell carcinomas. Hum Mol Genet. (2005) 14:603-14. doi: 10.1093/hmg/ddi057

Conflict of Interest: The authors declare that the research was conducted in the absence of any commercial or financial relationships that could be construed as a potential conflict of interest.

Copyright (๔ 2020 Wang, Zhu, Wang, Fan, Sun, Ji, Fan, Xie, Dai, Jin, Hu, Ma and Shen. This is an open-access article distributed under the terms of the Creative Commons Attribution License (CC BY). The use, distribution or reproduction in other forums is permitted, provided the original author(s) and the copyright owner(s) are credited and that the original publication in this journal is cited, in accordance with accepted academic practice. No use, distribution or reproduction is permitted which does not comply with these terms. 